\title{
Research on Magnetic Field Simulation of Cylindrical Linear Synchronous Motor
}

\author{
Jiang Minzheng ${ }^{1, *}$, Zhang Deshi ${ }^{1}$, Dong Kangxing ${ }^{1}$, and Wang Hui ${ }^{2}$ \\ ${ }^{1}$ School of Mechanical Science and Engineering, Northeast Petroleum University, Daqing, 163318 \\ ${ }^{2}$ Bomec Offshore Engineering Co., Ltd., Tianjin, 300457
}

\begin{abstract}
In order to obtain the influence of different structures and frequencies on the thrust of cylindrical permanent magnet linear synchronous motor, the transient simulation of magnetic field of cylindrical permanent magnet linear synchronous motor is carried out by using Ansoft software, and the correctness of the simulation is verified by experiments. Through simulation, the characteristics of the linear motor under different voltage frequency, air gap size, and permanent magnet width and its influence on the linear motor thrust are obtained.
\end{abstract}

\section{Introduction}

The cylindrical permanent magnet linear motor has the advantages of high thrust density, rapid dynamic response and reliable operation. It has been widely used in the field of high dynamic response servo and linear drive [1-3].

A large number of previous studies on cylindrical permanent magnet linear motor have been carried out. Through the combination of analytical method and finite element method, the theoretical and mathematical models of this kind of motor have been established, and verified by relevant experiments.Huang Kefeng and others explored the air gap magnetic density and waveform of cylindrical permanent magnet linear motor with each magnetizing structure, experimental results show that axial and Halbach magnetizing forms are characterized by large air-gap magnetic density, large range of variation and small effect of slotting [4].For the further improvement of thrust density of cylindrical permanent magnet linear motor, Luo Ciyong proposed a special-shaped permanent magnet Halbach array and established a magnetic circuit analysis model, the permanent magnet angle is obtained when the thrust density is maximum [5]. Cui Junfan et al discussed the influence of different pole-slot coordination and primary core length on the performance of the motor, which improved the sine of the air-gap magnetic density waveform of the fractional cylindrical permanent magnet linear motor and improved the thrust performance[6]. By means of finite element method and analytical method, the optimal design of magnetic field, back potential, thrust force, armature reaction, eddy current loss and position reduction of cylindrical structure motor is carried out by wangjiabin and so on[7].

Although some achievements have been made in the research of cylindrical permanent magnet linear synchronous motor, it is only based on theoretical research, lack of support of test data and test verification, and need to further improve the research to obtain accurate magnetic field characteristics. It lays a foundation for the research of cylindrical permanent magnet linear synchronous motor.

\section{Simulation of Transient Magnetic Field in Cylindrical Linear Synchronous Motor}

\subsection{Establishment of numerical model of motor}

The simulation model is drawn in the Cylindrical about $\mathrm{Z}$ coordinate plane by using the Transient module in the Ansoft according to the structural parameters of the actual cylindrical permanent magnet linear synchronous motor. This model includes Band for separating stationary parts from moving parts and Band in parts containing only moving parts, as shown in figure 1 .

Primary motor 45 steel, nonlinear material; Copper winding is used for primary winding, resistivity is (1), relative permeability (2); Secondary selection of ZG20 materials, permanent magnet N-S alternately placed between the oblique extremely fast; relative permeability (2).

$$
\begin{gathered}
\rho=2.17 \times 10^{8} \Omega \cdot \mathrm{m} \\
\mu_{r}=1
\end{gathered}
$$

The outer air boundary is set as the balloon boundary condition, the secondary part of the model is the moving part, the upward motion is defined as the positive direction, the synchronous motion speed is $0.198 \mathrm{~m} / \mathrm{s}$, the moving area is $0 \sim 400 \mathrm{~mm}$. The model of linear motor model is shown in figure 2.Set step size $0.001 \mathrm{~s}$, stop time $0.5 \mathrm{~s}$, a total of 500 steps.

\footnotetext{
* Author: Jiang Minzheng, male., Ph. D. supervisor, professor. E-mail:jmz1964@126.com
} 


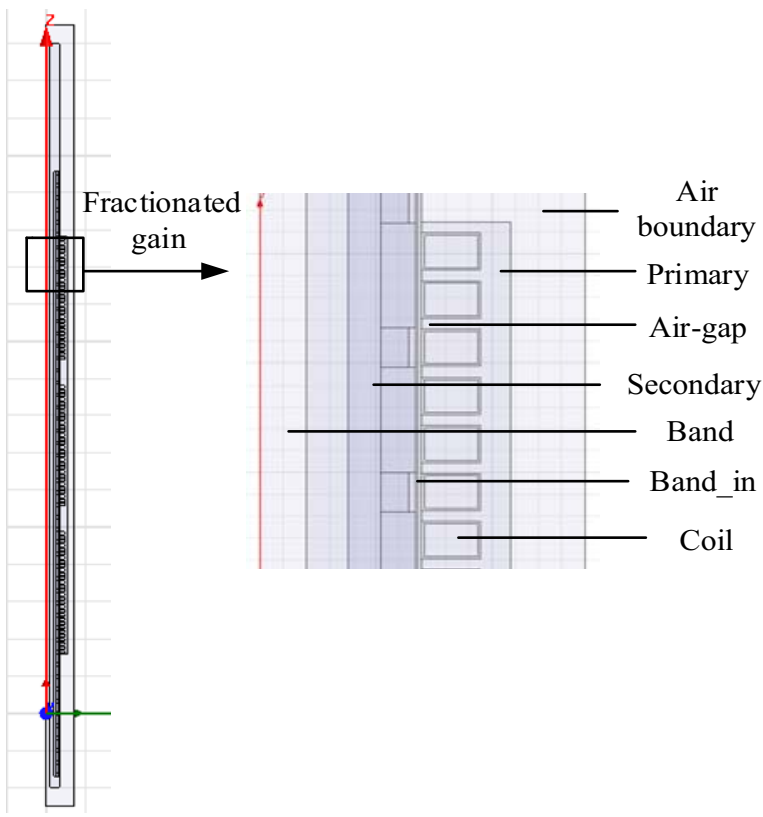

Fig. 1. Calculation model of cylindrical permanent magnet

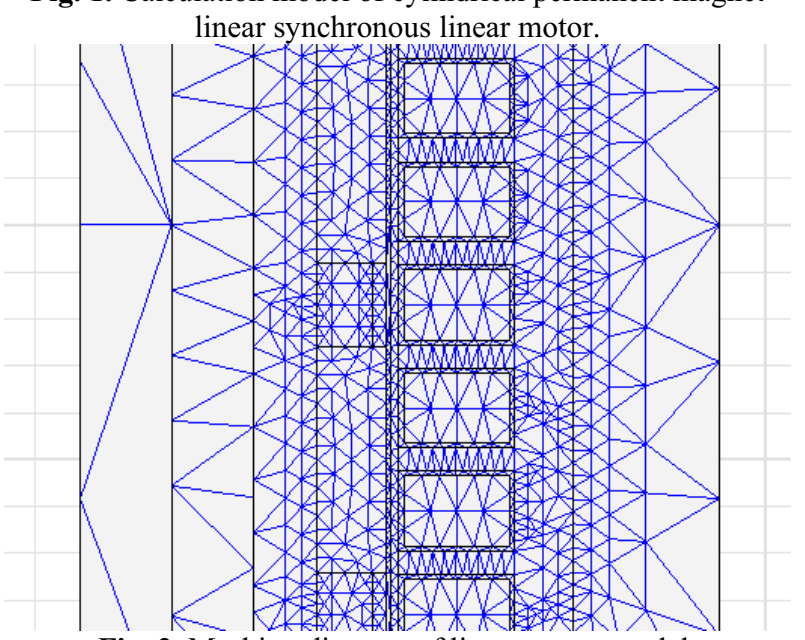

Fig. 2. Meshing diagram of linear motor model.

\section{Simulation Model of Motor Transient Magnetic Field}

To verify the correctness of the simulation model, using Ansoft software to simulate $5 \mathrm{~Hz} 、 4 \mathrm{~Hz} 、 3 \mathrm{~Hz}$ respectively, the transient characteristics of magnetic field in the stable operation of cylindrical permanent magnet linear motor with three different frequencies are obtained. The thrust curve of cylindrical permanent magnet linear synchronous motor with different frequencies is shown in figure 3.

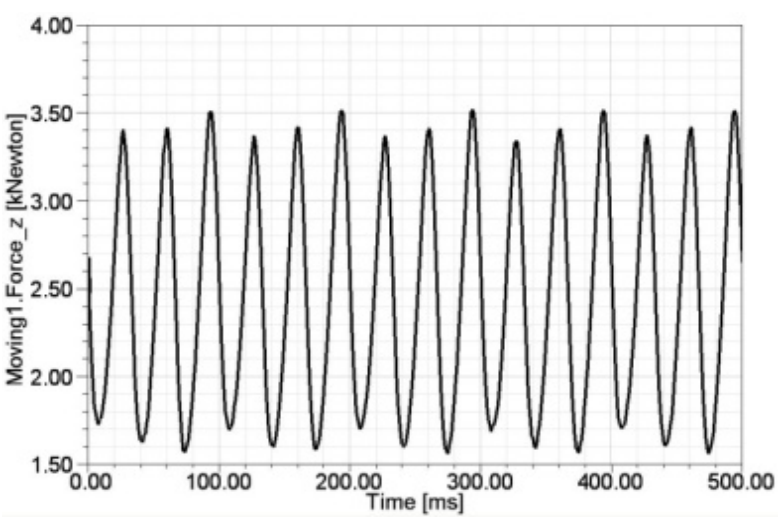

(A) $5 \mathrm{~Hz}$ motor running thrust curve

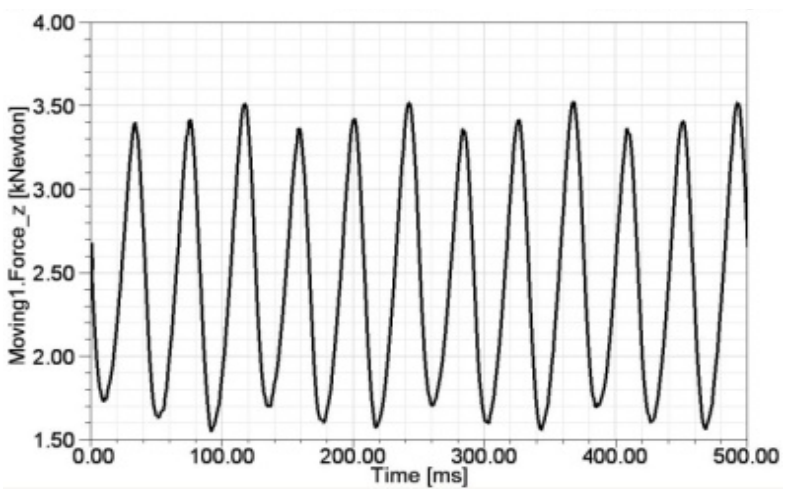

(B) $4 \mathrm{~Hz}$ motor running thrust curve

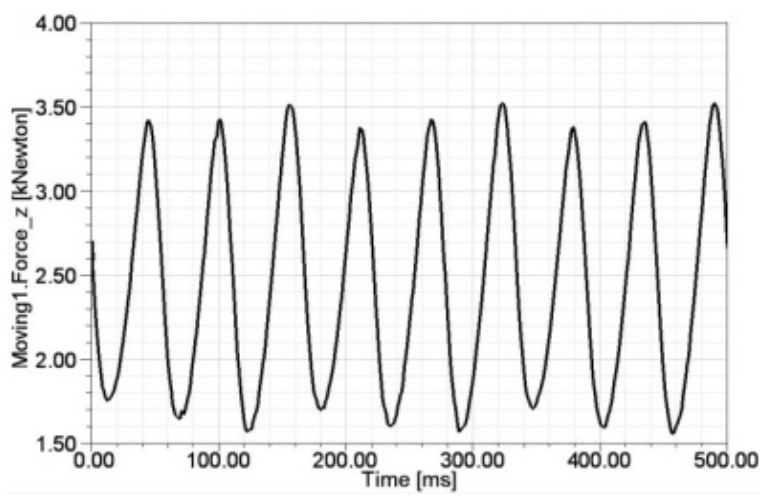

(C) $3 \mathrm{~Hz}$ motor running thrust curve

Fig. 3. Linear motor thrust curve at three frequencies

The thrust value of linear motor at different frequencies can be obtained from figure $3(\mathrm{~A}) \sim(\mathrm{C})$. The comparison with the thrust value obtained from the test is shown in Table 1

Table 1 Comparison of simulated thrust value and experimental thrust at different frequencies

\begin{tabular}{|c|c|c|c|}
\hline Frequency (Hz) & 5 & 4 & 3 \\
\hline $\begin{array}{c}\text { Simulated thrust } \\
\text { value (N) }\end{array}$ & 2525 & 2520 & 2550 \\
\hline $\begin{array}{c}\text { Test thrust value } \\
(\mathrm{N})\end{array}$ & 2474.38 & 2573.38 & 2581.452 \\
\hline Deviation (\%) & 2.04 & -2.08 & -1.22 \\
\hline
\end{tabular}

From Table 1, it can be seen that the error of using Ansoft software to simulate the thrust value of linear motor at different frequencies and the thrust value of 
linear motor obtained by the test are $2.04 \%,-2.08 \%$,$1.22 \%$, respectively, and the error value is small, which proves the feasibility of the established transient simulation of linear motor magnetic field.

\section{Analysis of electromagnetic field simulation results}

According to the simulation results, the transient characteristics of the linear motor under each parameter are analyzed and compared, and the influence of different parameters on the performance of the linear motor and its variation law are obtained.

(1)Effect of power frequency on thrust characteristics of linear motor

The three-phase AC frequency in the coil of cylindrical permanent magnet linear synchronous motor is $50 \mathrm{~Hz} 、 40 \mathrm{~Hz} 、 30 \mathrm{~Hz}$, respectively to keep the amplitude of three-phase AC voltage fixed at $250 \mathrm{~V}$. The electromagnetic thrust of cylindrical permanent magnet linear synchronous motor is simulated and simulated. The variation curve of secondary electromagnetic thrust with time at different frequencies is shown in figure 4.

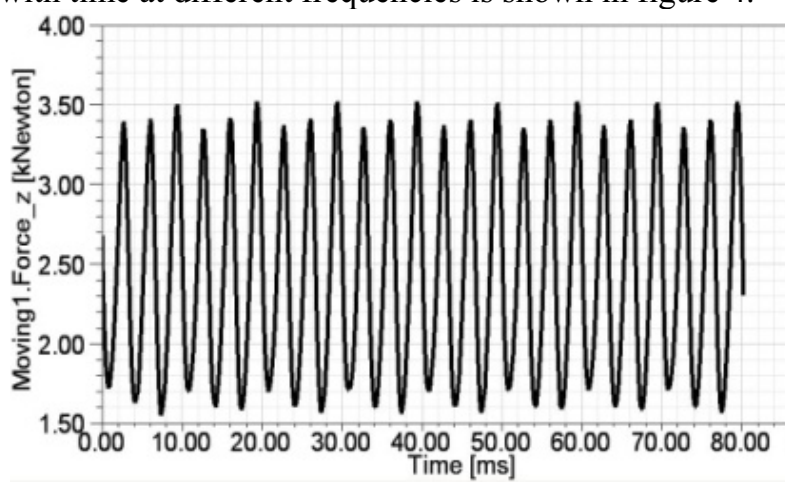

(A) $50 \mathrm{~Hz}$

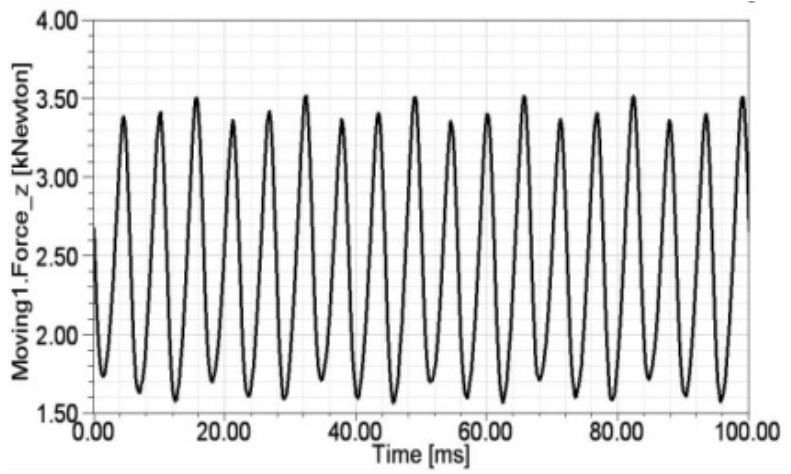

(B) $40 \mathrm{~Hz}$

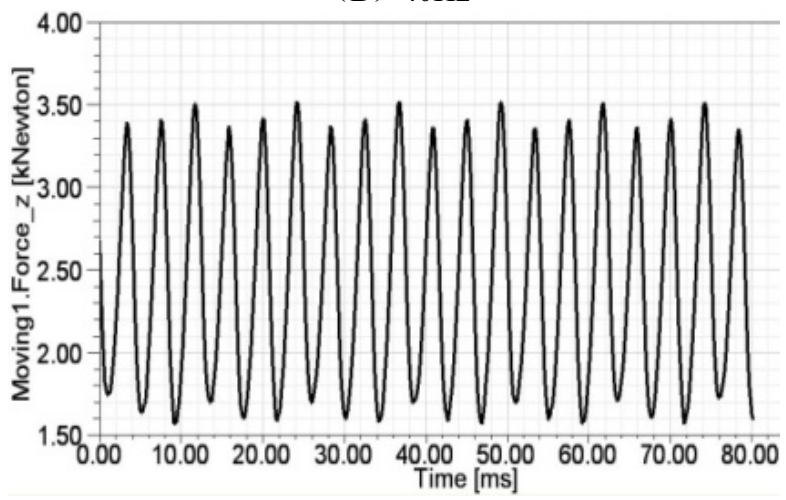

(C) $30 \mathrm{~Hz}$

Fig. 4. Motor thrust curves of three power supply frequencies varying with time

From the motor thrust curve at different power supply frequencies in figure 4 , when the amplitude of the power supply voltage of the linear motor is fixed and only the frequency of the power supply is changed, the electromagnetic thrust of the linear motor is constant, but the frequency increases gradually.

(2)Effect of air gap on thrust characteristics of linear motor

The air gap between the primary and secondary parts of the cylindrical permanent magnet linear motor is taken $2 \mathrm{~mm} 、 1.6 \mathrm{~mm} 、 1.2 \mathrm{~mm}$, respectively $\mathrm{mm}$ 、 $1.6 \mathrm{~mm} 、 1.2 \mathrm{~mm}$, and the amplitude of the phase voltage of the power supply is $250 \mathrm{~V}$, and the electromagnetic thrust of the linear motor is simulated when the frequency is 5. Figure 5 shows the thrust curve of different air gaps.

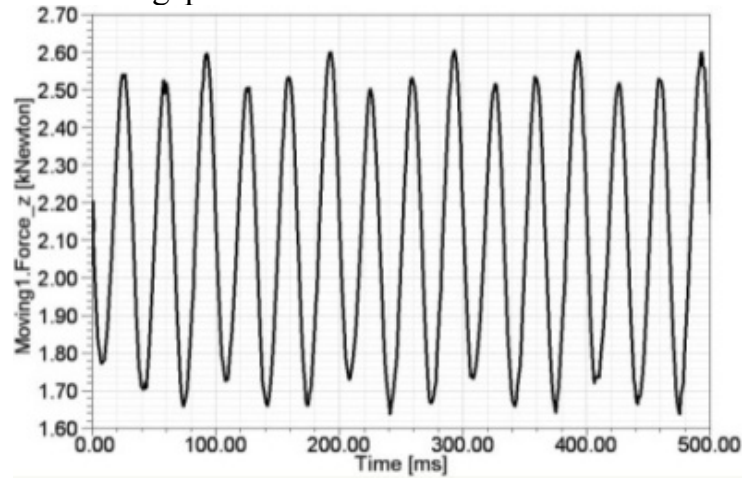

(A) $2 \mathrm{~mm}$

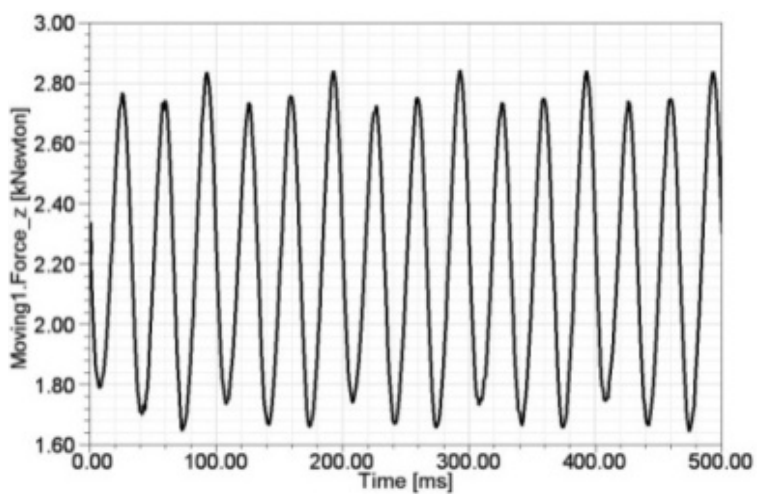

(B) $1.6 \mathrm{~mm}$

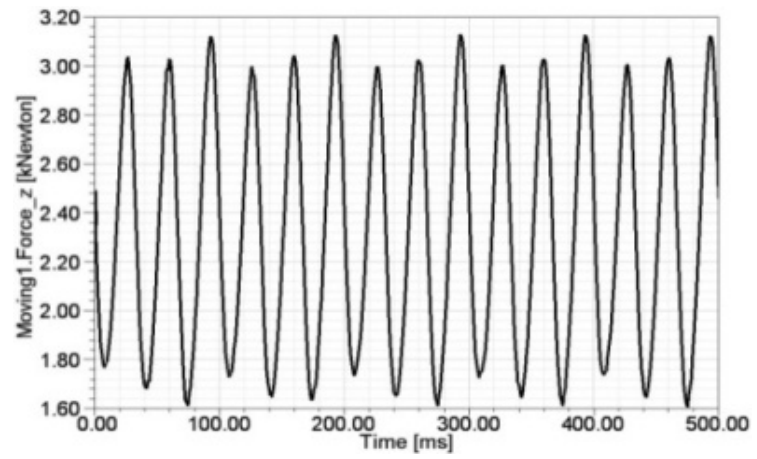

(C) $1.2 \mathrm{~mm}$

Fig. 5. Motor thrust curves of three types of air gaps with time 
The thrust curve of linear motor with different primary permanent magnet width in Fig .6 shows that the secondary permanent magnet width is $27 \mathrm{~mm}$, and the thrust of linear motor is $3900 \mathrm{~N}$; Secondary permanent magnet width $21 \mathrm{~mm}$, linear motor thrust $3350 \mathrm{~N}$; Secondary permanent magnet $15 \mathrm{~mm}$, linear motor thrust $3000 \mathrm{~N}$. Therefore, it can be concluded that as the size of the secondary permanent magnet becomes smaller, the thrust value becomes smaller, and its value decreases from $3900 \mathrm{~N}$ at $27 \mathrm{~mm}$ width to $3000 \mathrm{~N}$ at $15 \mathrm{~mm}$ width.

\section{Conclusion}

(1) A simulation model of transient magnetic field characteristics of cylindrical permanent magnet linear motor is established by using Ansoft software, and the correctness of the simulation model is verified by test results;

(2) With the change of running frequency of linear motor pumping pump system, the thrust value is constant and the fluctuation amplitude of thrust value is constant, but with the increase of frequency, the frequency of thrust fluctuation increases, and the thrust value becomes larger with the decrease of air gap.

\section{Acknowledgements}

The project is supported by Training plan for young innovative talents of University in Heilongjiang (UNPYSCT-2020150).

\section{References}

1. Xu Ying, Zhu Yuqiu. A Review of the Development of Bearingless Permanent Magnet Synchronous Motor System and Its Key Technologies $[\mathrm{J}]$. Journal of Electrical Engineering of China, 2019, 39 ( 10) : $2994-$ 3007 .

2. Li L, Pan D, Huang X. Analysis and optimization of iron less permanent-magnet linear motor for improving thrust $[\mathrm{J}]$. IEEE Transactions on Plasma Science, 2014, 41 ( 5 ) : $1188-1192$.

3. Lu Qinfen, Shen Jianming, Ye Yunyue - Research and Development of Permanent Magnet Linear Motor [J] . Journal of Electrical Engineering of China, 2019, 30 ( 9 ) : $2575-2587$.

4. Huang Kefeng, Li Huaishu, Zhou Yu , etc. Study on Air Gap Magnetic Field of cylindrical permanent magnet linear motor with different magnetizing modes $[\mathrm{J}]$. explosion proof motor, 2013, 48 (3): $1-4,9$.

5. Luo Ciyong, Li Zhutian, Shen Qiping. Optimal design of cylindrical linear motor with special-shaped permanent magnet [ J ] . Journal of Electrical Technology, $2017,32(17): 127-134$.

6. Cui Jiefan, Li Yi, Liu Xu. Design and finite element analysis of cylindrical permanent magnet linear motor $[\mathrm{J}]$. print motor, 2016, 49( 5) : $25-27$.

7. Wang Jiabin, Howe David. Tubular modular permanent magnet machines equipped with quasi halbach magnetized magnets Part I: Magnetic field distribution, EMF and thrust $[\mathrm{J}]$. IEEE Trans. on Magnetics, 2005, 41( 9) : 2470 2478.

8. Wang Jiabin, Howe David. Tubular modular permanent magnet machines equipped with quasi halbach magnetized magnets part II: magnetic field distribution, EMF, and thrust force [ J ] IEEE Transactions on Magnetics, 2005, 41 ( 9) : $2479-2489$.

9. Zhao Jinghong, Zhang Xiaofeng, Zhang Junhong, etc. Magnetic field and thrust analysis of cylindrical permanent magnet linear synchronous motor $[\mathrm{J}]$. Journal of Motor and Control, 2010, 14 ( 1 ) : 12-17.

10. Liu Xingqiao, Huang Xinguang, Wang Yan, etc . Analysis of winding of cylindrical permanent magnet synchronous linear motor [J] . Electrical and Control Applications, 2013, 40(3):8-12.

11. Cui Jiefan, Qin Chao, Wang Hongxue. Analysis on Minimization of Magnetoresistive Force in Cylindrical Permanent Magnet Linear Motor [J] . Combined Machine Tool and Automation Machining Technology, 2014 ( 6 ) : 25 - 27. 